213. Erik Hornung, Das Amduat. Die Schrift des verborgenen Raumes. Hrsg. nach Texten aus den Gräbern des Neuen Reiches I: Text, II : t'bersetzung und Kommentar. Wiesbaden $1963=$ ÄgAbh 7 (ThLZ 90, 1965, 501-503).

214. Eberhard Otto, Gott und Mensch nach den Tempelinschriften der griechisch-römischen Zeit. Eine Untersuchung zur Phraseologie der Tempelinschriften, Heidelberg $1964=$ Abhandl. d. Heidelberger Akad. d. Wiss., Phil.-hist. Kl. 1964, 1 (ThLZ 90, 1965, 669-673).

215. Annales du Service des Antiquités de l'Égypte 58, 1964 (und Mitarbeiter, Zeitungsschau, in: OLZ 61, 1966, 202-204).

216. Alexandre Piankoff, The Litany of Re, New York $1964=$ Pantheon Books. Bollingen Series XL, Egyptian Religious Texts and Representations 4 (Bibliotheca Orientalis 25, 1968, 181-183).

217. Bulletin de l'Institut français d'archéologie orientale 61, 1962 (und Mitarbeiter, Zeitungsschau, in: OLZ $63,1968,613-614)$.

218. Erik Iversen, The Myth of Egypt and its Hieroglyphics in European Tradition, Copenhagen 1961 (OLZ $63,1968,130-131$ ).

219. Otto Kaiser, Israel und Ägypten. Die politischen und kulturellen Beziehungen zwischen dem Volk der Bibel und dem Land der Pharaonen = Vortrag Hildesheim Roemer-Pelizaeus-Museum 1963, Zeitschr، d. Museums zu Hildesheim N.F. 14, 1963 (OLZ 63, 1968, 354-356).

220. Erik Hornung, Das Amduat. Die Schrift des verborgenen Raumes. Hrsg. nach Texten aus den Gräbern des Neuen Reiches. III. Die Kurzfassung. Nachträge, Wiesbaden $1967=$ ÄgAbh 13 (ThLZ 93, 1968, 414-417).

221. Fritz u. Ursula Hintze, Alte Kulturen im Sudan, Leipzig 1966 (Ethnographisch-archäologische Zeitschr. 10, 1969, 572-573).

Siegrried Morenz $\dagger$

\title{
Traditionen um Menes
}

\section{Beiträge zur überlieferungsgeschichtlichen Methode in der Ägyptologie II*}

Unter den Verdiensten, die sich W. Spiegelberg um die Ägyptologie erworben hat, sind seine Studien über Herodots Verhältnis zu Ägypten ${ }^{1}$ nicht die kleinsten. Diese „schönste Würdigung Herodots von ägyptologischer Seite“ "2 läßt einen gesunden Sinn ihres Verfassers für „Uberlieferungsgeschichte als Problem" ahnen, die dem Erforscher ägyptischer Historie heute aufgegeben ist $^{3}$. So scheint es mir angebracht, in dieser Gedenkschrift davon eine Probe zu geben. Sie gilt zugleich dem Meister der Erforschung ägyptischer Spätzeit. Dabei löst sich die scheinbare Paradoxie, ausgerechnet den von der Tradition erkorenen Stifter der ägyptischen Geschichte mit dem Ausgang ihres Laufes zu verbinden, durch das Vehikel der Überlieferung wie von selbst auf. Mit alledem wird von vornherein deutlich gemacht, worum es hier geht: Es ist die außergewöhnliche Rolle der Tradition, die über den Anfang der ägyptischen Geschichte, Landschaft und Kultur einschließend, an ihrem Ende immer dichter gewoben wurde.

* Der erste Teil dieser Beiträge erschien unter dem Titel „Traditionen um Cheops “ in ZÄS 97, 1971, S.111ff. - Der vorliegende Artikel war ursprünglich für die Gedenkschrift W. Spiegelberg konzipiert. S. Morenz hat bis zu seinem letzten Lebenstag daran gearbeitet, konnte ihn aber nicht mehr vollenden. Dennoch halten wir die Veröffentlichung für sinnvoll, zumal das Anliegen schon im ersten Aufsatz zur überlieferungsgeschichtlichen Methode dargestellt worden ist. Aus Gründen der Pietät wurde auf eine Zusammenfassung seiner Ausführungen verzichtet. Als Fragment aber konnten sie nur in der Gedenkschrift für den verstorbenen Verfasser Platz finden. Trotzdem wurden die Bezüge auf die Spiegelberg-Gedenkschrift beibehalten. Die Red.

1 Die Glaubwürdigkeit von Herodots Bericht über Ägypten im Lichte der ägyptischen Denkmäler, 1926.

2 M. Kaiser bei S. Morenz, Die Begegnung Europas mit Ägypten², 1969, S. 271 (= A. 8).

3 ZÄS 97, 1971, S. 112. 
Die Methode, deren man sich dabei bedienen muß, haben wir in dem eingangs zitierten Beitrag „Traditionen um Cheops" gekennzeichnet und dürfen jetzt darauf verweisen. Zu den dort verzeichneten Schichten, aus denen sich das Gebäude einer Überlieferung aufbaut und die für Altägypten relevant sind, tritt im Falle des Menes eine weitere, die sich durch das Gegensatzpaar Geschichte : Mythos kennzeichnen läßt‘. Dieses Plus zum Schichtenkomplex bei Cheops ist deutlion qualitativer Natur. Im Unterschied zu den Paaren mündlich : schriftlich, offiziell : inoffiziell, inländisch : ausländisch usw. stellt es die Historizität selbst in Frage. Eben damit steigt aber die Macht der Ideologie empor, die sich äußerstenfalls in der Lage zeigt, „Fakten“ durch Utberlieferung zu schaffen. Tatsächlich bestehen auch hier die wesentlichen Unterschiede in Hinsicht auf Cheops und Menes. An der Cheopspyramide läßt sich nicht rütteln, die allfälligen kümmerlichen Spuren des Menes aus seiner Zeit hingegen können ebensogut bestritten wie behauptet werden. Das ist zunächst zu vergegenwärtigen.

Wie im Falle der Überlieferung um Cheops gehen wir auch diesmal von der Arbeit aus, die am letzten Wirkungsorte Spiegelbergs, also im Münchner Ågyptologischen Seminar, entstanden ist und vom Material her die Tore zu allen einschlägigen Forschungen eröffnet hat ${ }^{5}$. Bei der dortigen kritischen Sichtung möglicher zeitgenössischer Zeugen ist nur einer übrig geblieben: der durch eine Abrollung erschließbare Siegelzylinder aus Abydos mit dem Zeichen שس neben dem in das Bild des „Horus-Palastes“ eingeschriebenen Namen $N^{c} r-m r^{6}$. Gegenüber der älteren Naivität, die unbeschadet der vielen Funktionsmöglichkeiten des Zeichens بس auf den Königsnamen Menes schließt 7, hat die Forschung also inzwischen eine äußerste Reduktion vorgenommen. Man war vor der hier zugrunde gelegten jüngsten Arbeit freilich sogar noch einen Schritt weitergegangen und hatte Menes für eine fiktive Person erklärt, deren Name aus dem Wort $m n$ „Irgendwer" entstanden sei ${ }^{8}$. Aber selbst die Konservatoren des letzten Belegstückes räumen ein, daß dieser archaische Menes, wenn mit dem vieldeutigen Zeichen ein Mann dieses Namens gemeint ist, nur ein Funktionär und d. h. damals wohl soviel wie ein Prinz (aber keineswegs unbedingt ein Thronanwärter) und jedenfalls kein König gewesen ist ${ }^{9}$. Wäre es so - und die Möglichkeit müssen wir einräumen - könnte der große Menes zufolge der Siegelabrollung als Sohn des Narmer nur mit dessen Nachfolger geglichen werden, also mit dem Träger des Horusnamens ' $h 3^{10}$. Damit kommt man indes historisch in die Verlegenheit, den „Reichseiniger" nicht mit seinen starken Vorgängern „Skorpion“ und $N^{c} r$ - $m r$ identifizieren zu können 11. So erhebt sich zwangsläufig die Frage, warum man später die entscheidenden Leistungen einem Manne zugeschrieben haben soll, der in Wirklichkeit vielleicht nur ein weniger bedeutender Zweiter oder Dritter in der Reihe gewesen ist ${ }^{12}$.

Angesichts des dargelegten Befundes scheint mir indes die Tragfähigkeit des Materials überhaupt bei weitem überfordert, wenn man die Existenz eines Königs Menes aus zeitgenössischen Quellen erweisen will. Ich kehre daher den Spieß um und frage: Wäre jemand auf den Gedanken

4 Etwa im Sinne von P. Grimal, Der Mensch und der Mythos, in: Mythen der Völker, 1967, bes. S. 20: „Daß der Troianische Krieg vielleicht gar nicht stattgefunden hat, ist völlig unwichtig“ - d. h. wesentlich war seine „mythische Realität", die für die Hellenen unablässig ihre Wirkung tat.

5 D. Wildung, Die Rolle ägyptischer Könige im Bewußtsein ihrer Nachwelt I = MÄS 17, 1969. Zeugnisse zu Menes gesammelt und kommentiert S. $4 \mathrm{ff}$.

6 Wildung, a. a. O. S. 4 nach W. M. F. Petrie, Royal Tombs II, 1901, S. 30 und T. 13 , Nr. 93.

7 So Ed. Meyer, Geschichte des Altertums I 22, 1909, S. 209 und seine Gewährsleute.

8 Eingeschrieben in Ritualtexte und als Königsname verstanden: Ph. Derch ain, Ménès, le roi „Qelqu'un ", in: RdE 18, 1966, S. $31 \mathrm{ff}$; abgehandelt von Wildung, a. a. O. S. 5.

9 Zu den „Prinzensiegeln“ vgl. W. Helck, Gab es einen König Menes? in: ZDMG 103, 1953, S. 354ff., zur Rolle der Prinzen als Funktionäre: ders., Untersuchungen zu den Beamtentiteln des Alten Reiches $=$ ÄgFo 18, 1954, bes. S. 11 f. und 15ff., P. Kaplony, Inschriften der ägyptischen Frühzeit I, 1963 , S. 71 f.

10 Diese Konsequenz zieht in der Tat Wildung, a. a. O. S. 5.

11 Wildung gerät daher prompt ins Gedränge: S. $28 \mathrm{f}$.

12 J.Ph. Lauer, Quelques remarques sur la Ie dynastie, in: BIFAO 64, 1966, S. 169ff. hat vor allem deshalb für die Identität des Menes mit Narmer plädiert und zugleich die von Vorgängern erwogene Möglichkeit zurückgedrängt, daß Menes eine Art Sammel-,Ehren“-Name für die Gründerkönige gewesen sei. 
gekommen, diesen König als historische Figur in frühzeitlichen Zeugnissen um jeden Preis aufzusuchen, wenn eine sehr viel spätere Überlieferung ihn nicht namhaft machen würde? Damit stelle ich die erste und grundsätzliche Entscheidung der überlieferungsgeschichtlichen Macht anheim. Sollte sich zeigen, daß daraus die Kreierung eines mit dem wahrhaft redenden Namen $M n j$,der Bleibende" durch die Tradition abgeleitet werden kann, sieht die Sache natürlich ganz anders aus. Die etwas peinliche Frage lautet nachher: Wie kann man eine Schöpfung der Tradition ,in der Wirklichkeit" finden wollen? Jedenfalls wird man erst nach Absolvierung dieses bisher unterlassenen Arbeitsganges über die derzeitigen reinen Ermessensurteile hinauskommen. Ansätze in der Forschung sind da. Sie müssen freilich substantiell ausgebaut und in die methodische Klarheit geführt werden.

Doch zunächst haben wir uns in Erinnerung zu rufen, wie es mit der Überlieferung von Menes steht. Wann setzt sie ein, wie sieht sie aus, wo treffen wir sie an? Das Material ist im wesentlichen gesammelt ${ }^{13}$. Es setzt mit einem Fortissimo eil, aber erst in der frühen Ramessidenzeit. Der Klang ist in den Königslisten zu Abydos noch halbwegs verhalten. Immerhin erscheint Menes hier als Erster in der langen, prinzipiell lückenlosen Kette ägyptischer Könige bis zu Sethos I. bzw. Ramses II. Der Gedanke an den Gründer oder Stifter einer großen Institution läßt sich kaum überhören. Ihren vollen Ton erreicht die Aussage jedoch erst im Turiner Königspapyrus (Ramses II.). Jetzt wird Menes, um in der einmal gewählten Bildrede zu bleiben, zum Grundton des historischen Königtums. Sein Name schließt die mythische Periode der $3 h w$ und $s m s w ~ H r$ im Kontext einer Summierung ab: „Summe ihrer Jahre bis hin zu dem König von Ober- und Unterägypten $M n j$, der lebt, heil und gesund ist" (col II 1,20). Darauf (II 1,11) beginnt die Reihe der "normalen, menschlichen" Herrscher mit $M n j$. Aber noch zweimal bezeichnet $M n j$ Abschnitte innerhalb ihrer Folge. Anfangs wird eine „Summe der Königsherrschaften von $\mathbf{M} n j$ bis Wnjs" gezogen (III 1,26). Später (IV 1,16) faßt man von ihm an bis zu einem Punkt nach dem Ende der sechsten Dynastie das ganze Alte Reich zusammen ${ }^{14}$. Von zweifacher Bedeutung ist daneben die Königsliste im Totentempel Ramses' II. (Ramesseum). Einmal läßt sie eine einleuchtende und klare Gliederung der ägyptischen Geschichte erscheinen. Denn sie stellt Menes und Mentuhotep ( $N b-h p . t-R^{*}$ ) als Repräsentanten der ersten und zweiten historischen Phase neben die (rückwärts abgespulte) Kette der Herrscher des Neuen Reiches von Ahmose bis Ramses II. Menes war also stark genug, gleich einer mächtigen Säule das eine Drittel der dreischiffigen Halle ägyptischer Geschichte allein zu tragen. Er stellt sich, wie Mentuhotep, als Symbolfigur dar: nicht „unhistorisch“, eher „überwirklich“ ${ }^{15}$. Zum anderen baut sich die Ramesseumsliste auf einem Geleit auf, das die genannten Könige im Gefolge des Gottes Min bei seinem großen Fest zeigt 16. Dieser damit gesetzte Zusammenhang wird uns noch gründlich beschäftigen. Einstweilen merken wir ihn lediglich vor. Aus der gleichen Zeit stammt eine Berliner Schreibpalette, deren (thebanischer) Inhaber Imn-w3h-sw u. a. den „Ptah des $M n-n 3^{\prime \prime}$ in die Totenopferformel einbezieht. In diesem $M n-n 3$ hat man seit langem den Namen des Menes gesehen. Die Fügung selbst ist auf Grund vieler Parallelen als Bezeichnung eines bestimmten Ptahtempels gedeutet worden, den man auf Menes zurückführte ${ }^{17}$. Das kann nicht überraschen, wo doch Menes damals als Gründer und Stifter der ägyptischen Geschichte galt und sein Name in der Überlieferung so stark zum Klingen kam, wie das in den offiziellen historischen Texten der Zeit geschah. Was diese betrifft,

13 Wildung, a. a. O. S. $6 \mathrm{ff}$.

11 Es scheint mir bisher nicht geglückt zu sein, die Absicht gerade dieser Einteilung zu ermitteln. H. Ranke, Vom Geschichtsbilde der alten Ägypter, in: CdE 6 (12), 1931, S. $277 \mathrm{ff}$. nennt sie schlicht „selt. sam" (S. 281).

15 Wildung möchte (S. 12), daß man den Grund „historischer Leistungen“ für die Nennung von Menes und Mentuhotep nicht überbewerte. Ich spreche mit Bedacht von „Symbolfiguren“, um einerseits den Weg für die „Nicht-Existenz“ des Menes völlig offenzuhalten, anderseits die mit der Parallelgestalt des Mentuhotep im wahrsten Sinne des Wortes ,tatsächliche“ historische Bedeutung nicht zu verkürzen.

${ }^{16} \mathrm{Zu}$ diesem u. a. C. J. Bleeker, Die Geburt eines Gottes, 1956, S. $69 \mathrm{ff}$.

17 Erörterung und Literatur (seit A. Erman, in: ZÄS 30, 1892, S. 44 ff.) bei Wildung, a. a. O. S. 12 ff., der in plausibler Weise auch eine genetische Verbindung zur Ramesseumsliste herzustellen sucht. 
so darf man von einer „vertikalen Ideologie“ sprechen, die in der Ramessidenzeit das ägyptische Königtum geistig nicht nur in der Breite des Raumes entfaltet, sondern auch in den Tiefen der Zeit verankert hat ${ }^{18}$.

Damit verschwinden die ägyptischen Zeugnisse für Menes auf lange Zeit. Die nächsten, nach Inhalt und Umfang ergiebigeren Aussagen macht uns Herodot, der damit den Zweig ausländischer Überlieferung beginnt. Da sie aber in besonderer Weise aufbereitet werden muß, setzen wir zunächst die Vergegenwärtigung der restlichen ägyptischen Dokumentationen fort. Da ist zunächst eine Stele aus dem Serapeum, deren Stifter $N \dot{s}$-nww-wr sich etwa in die Mitte des 2 . Jahrhunderts datieren läßt. Natürlich spielt Apis im Bildfeld, aber auch in den Anreden eine beherrschende Rolle. Wir werden uns dessen zu erinnern haben. Menes erscheint in den Ämtern, die der Vater des Stifters namens Wnn-nfr bekleidet hat. Er war Priester der Könige Nektanebos (Nht- $H r-h b j t)$, Menes und Ttj. Nektanebos, damals rund zweihundert Jahre tot, wurde bis tief in die Ptolemäerzeit vor allem als Bauherr verehrt ${ }^{19}$. Zu Ttj kann ich en passant nichts sagen ${ }^{20}$. Was aber Menes betrifft, so ist vorab an die Rolle von Memphis zu denken, das mit der Namensvariante im Kontext erscheint. Es liegt natürlich nahe, bei Apis an „Menes und die Gründung von Memphis“ zu denken ${ }^{21}$. Auf die Beziehungen, die die Tradition anderwärts zwischen Apis und Menes herstellt, sei jetzt nur vorausgewiesen. Wahrscheinlich ebenfalls aus dem Raum Memphis (Saqqara) stammt der etwa gleichzeitige Berliner Sarg des Snbf. Der Tote war u. a. Priester des Menes und des Zoser, also zweier Könige, über deren von Überlieferung bzw. Geschichte hergestellte Verbindung zu Memphis kein Wort verloren zu werden braucht.

Jetzt dürfen die Wasser der griechischen (und lateinischen) Überlieferung in das reichlich trockene Bett der ägyptischen Tradition um Menes eingelassen werden. Mit Herodot (II 4 und 99) beginnend, sind sie mindestens über die Ptah-Priester von Memphis an diese Stromführung legitim angeschlossen. Es scheint am zweckmäßigsten, mit dem Motiv des Finders, Gründers oder auch Schöpfers zu beginnen ${ }^{22}$. Man darf da freilich, wie sich sogleich herausstellen wird, bei Herodot nicht stehenbleiben. Ihm zufolge hat Menes, im Sinne der mythischen Vorstellung vom Urhügel ${ }^{23}$, unterhalb des „Moirissees“ (Faijûm) durch Abdämmung festes Land geschaffen und darauf Memphis gegründet. Es mag schon hier auf allfällige historische Hintergründe sowohl wie Beziehungen des Menes zum Moírissee ${ }^{24}$ in der späteren Überlieferung hingewiesen werden. Gründung von Ortschaften durch Kanalisation ist schon in der ersten Frühzeit faßbar, und gerade der Name der alten Faijûmstadt $\breve{S} d t$ (Krokodilopolis) bedeutet etwa „durch Graben gebildetes Land" 25 . Griechische Überlieferung aber läßt Menes als Gründer von Krokodilopolis erscheinen. Sie verbindet damit jene Geschichte von der Verfolgung des Königs durch seine Hunde, seine Flucht in den Moirissee und seine Rettung durch ein Krokodil, dessen Traditionsspuren man in die neuägyptische Erzählung vom verwünschten Prinzen zurückverfolgen mag ${ }^{26}$. Wir kommen darauf noch einmal zurück und runden zunächst das Bild von Menes dem Finder, Gründer und Schöpfer ab. Dabei entspricht das Alter der Zeugen etwa dem Gewicht der Sachen, so daß beide gemeinsam die

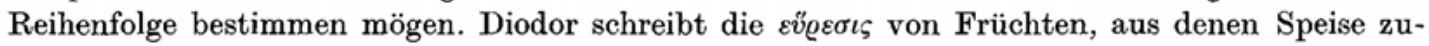

18 Es kann hier nicht erörtert werden, was das für das Geschichtsverständnis der Ramessidon bedoutet vgl. das entsprechende Problem in ZÄS 97, 1971 , S. 117.

$19 \mathrm{Zu}$ allem Vorstehenden Wildung, a. a. O. S. $15 \mathrm{f}$.

20 Soweit nicht bei Wildung, a. a. O. S. 18: $\underline{D} s r$ und ( $\underline{D} s r)-T t j$ und S. 20 Hinweise gesehen werden.

21 K. Sethe, in: UGAÄ 3, 1905, S. 121 im Anschluß an Herodot II 99 nach Zeugnis der Priester des Ptahtempels.

22 Es ist von H. Brunner, Menes als Schöpfer, in: ZDMG 103, 1953, S. 22 ff. artikuliert worden.

23 Brunner, a. a. O. S. $24 \mathrm{ff}$.

24 Diodor I 44 nennt lt. zwingender Konjektur den ersten König „Moiris“, s. u. A. 50.

25 P. Kaplony, in: CdE 44, 1969, S. 56. Die Stadt wird durch ein Siegel des Narmer bezeugt, vgl. H. Bonnet, RÄRG, 1952 , S. 394 .

26 Diodor I 89, dazu G. Posener, On the Tale of the Doomed Prince, in: JEA 39, 1953, S. 107; daß ,in Wirklichkeit" sicher eine Kontamination mit Amenemhet III. (Lamares, Labyrinth) vorliegt, interessiert uns hier nicht. 
bereitet werden kann, alternativ zur Kulturheroine Isis „einem der alten Könige mit Namen Menes" zu ${ }^{27}$. Der ältere Plinius nimmt in seine Naturgeschichte einen Zeugen auf, demzufolge Menes ,in Ägypten die Buchstaben erfunden habe“ "28. Später findet sich in Aelians Werk über die Natur der Tiere die Verehrung des Apis auf Menes zurückgeführt 29. Diese Spezialität lehnt sich vermutlich an die Tradition um Memphis und Menes an. Sie fordert aber im besonderen den Blick auf das obengenannte ägyptische Denkmal, in dem ein ptolemäischer Menespriester ausgerechnet auf einer Apisstele erscheint. Auch erinnert sie an den Gründer eines Ptahtempels, waren doch die Memphiten Apis und Ptah eng verbunden. Nehmen wir diese z. T. ja ganz beiläufig gemachten Aussagen antiker Autoren zusammen, so ergeben sie ein eindrucksvoll reiches Bild von Menes als Kultheros, der demzufolge nicht zufällig mit Isis konkurriert. Man kann es im Stile einer Aretalogie beschreiben. Er hat Memphis gegründet ${ }^{30}$. Er hat der Landschaft unterhalb des Faijûm ${ }^{31}$ das sie fortan kennzeichnende Gepräge gegeben. Er hat den Ägyptern die Nahrung oberhalb primitiver Stufen erschlossen. Er hat ihnen die Schrift erfunden. Er hat einen Kult eingesetzt. Er hat einen Tempel gegründet. Diese Traditionen kann man nur bedingt ,ausländisch" nennen. Sie gehen im Falle Herodots bestimmt, im übrigen mutmaßlich auf späte Überlieferungen im Lande selbst zurück. Insgesamt aber sind sie vergesellschaftet mit dem, was die offiziellen ägyptischen Listen aussagen: daß Menes der erste menschliche König auf dem Thron des Horus gewesen ist. Um die Aretalogie abzurunden: Er hat das Königtum von den Göttern und Heroen auf Menschen übertragen. Im Grunde entfalten also die antiken Quellen nur die ganze Fülle der Möglichkeiten, die dem ägyptischen König kraft der angestammten Ideologie zukommt.

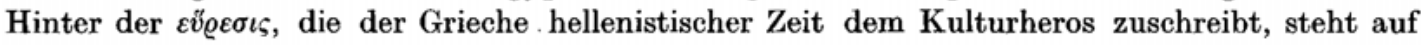
ägyptischer Seite nicht zuletzt das „Anfangen“ einer Tätigkeit, das seinerseits mit dem schöpferischen Akt des „ersten Males“ zusammenhängt ${ }^{32}$. Der allmächtige Pharao klassischer Zeiten Ägyptens wird zum Kulturheros hellenistischen Gepräges weiterentwickelt. Mit dieser Einsicht ausgerüstet müssen wir jetzt eine kardinale Frage nach rückwärts in die ägyptische Geschichte hinauf richten. Sie lautet: Hat die Ideologie schon in den offiziellen Zeugnissen der Ramessidenzeit an der Gründergestalt gebaut? Für eine Antwort gehen wir vom Namen des Helden aus und werden uns auf diesem Wege sogleich einer doppelgleisigen Überlieferung gegenüber sehen.

Zuerst mag die uns geläufige Form behandelt werden, also $M n j$. Griechisch im allgemeinen $M \eta \dot{v} \eta \varsigma^{\prime}$, lateinisch Menes. Der ägyptische Name bedeutet bekanntlich den „Bleibenden“, „Dauernden". Es liegt auf der Hand, daß er wie geschaffen scheint für einen Mann, der von der Überlieferung als Erster in der Reihe des Königtums herausgestellt wird. Der Bleibende und Dauernde gewinnt dabei leicht den Sinn des „Beständigen“. Das ist um so gravierender, als zwei mythische Modelle die Beständigkeit der königlichen Ausgangsposition darstellen. Erstens ist nach ägyptischer Überzeugung jeder König als Horus Sohn des Osiris, und der anfängliche König Osiris nimmt jeden irdischen Herrscher nach dem Tode wieder in sich auf. Der anfängliche König bleibt also bestehen. Zweitens ist der Kamutef im Spiele. Dieser "Stier seiner Mutter" ist Vater und erzeugt sich als Sohn selbst, wobei Vater und Sohn im stets wiederholten Vorgang dauerhafte Größen sind ${ }^{33}$. Wir halten einstweilen fest, daß der Kamutef vor allem mit dem Gotte Min verbunden worden ist, der uns nächst Osiris gründlich beschäftigen wird. Wer die Wachheit des

27 I 43. Es handelt sich vorab um das bereits I 34 als "Grundnahrungsmittel" hervorgehobene Lotos. brot, zu dessen mythischen Bezug man Morenz-Schubert, Der Gott auf der Blume, 1954, S. 77f. vergleiche.

${ }_{28}$ VII $56=$ Hopfner, Fontes S. 192.

${ }_{29} \mathrm{XI} 10=$ Hopfner, Fontes S. 424, dazu E. Otto, Beiträge zur Geschichte der Stierkulte in Ägypten $=$ UGAÄ 13, 1938, S. 5 mit A. 2 und S. 11 (über das hohe Alter des Kultes).

30 Das als $h t-k 3-P t h=A$ ĭ $\gamma v \pi \tau o \varsigma$ für Viele mit „Ägypten“ identisch war.

31 Wo ihn zudem Lokaltraditionen erfassen.

32 Dazu D. Müller, Ägypten und die griechischen Isisaretalogien = ASAW 53, 1, 1961, S. 22ff.

33 Im Hintergrund steht die Idee der „Dynastie“ (besser: Königsreihe), auf die H. Jacobson, Die dog. matische Stellung des Königs in der Theologie der alten Ägypter $=$ ÄgFo 8, 1939, bes. S. 28ff. in ihrer Verbindung mit dem Kamutef (vorab als Min) hingewiesen hat. Ich akzeptiere das, ohne dem Vf. in andere Konsequenzen zu folgen. 
Agypters für den Zusammenhang von Sache und Wort bedenkt, der u. a. so viele Wortspiele zuzuschreiben sind ${ }^{34}$, wird nicht bezweifeln, daß für ihn gerade im Falle des Menes dieser Sachbezug eines Wortes von größter Bedeutung gewesen ist. Die Griechen haben das augenscheinlich gewußt. Jedenfalls setzt Eratosthenes den Aićvı Königslisten ${ }^{35}$. Angesichts dieses klaren Beleges kann man sich absichernde Konstruktionen sparen, die etwa bei dem weitgehenden Gleichklang des ägyptischen mit dem griechischen Worte für „bleiben“ anzusetzen hätten: $m n$ und $\mu \varepsilon ́ v \varepsilon \iota v$. Sie wären leicht weiterzuführen über die Inschrift eines Aionbildes aus Eleusis, die $A i \omega ́ v$ und $\mu \varepsilon \dot{v} \omega v$ in eine griechische Fügung nimmt ${ }^{36}$. Auch denkt man an die rekonstruierbare Wiedergabe von $(d j){ }^{~} n h \underline{d} d t$ durch ai $\omega v o ́$ $\beta \iota \iota$ in der „Obeliskenübersetzung des Hermapion" ${ }^{37}$, wodurch wiederum eine Wurzel in diesem Prädikat für ptolemäische Herrscher freigelegt wird ${ }^{38}$. Wichtiger für unseren Zusammenhang ist etwas anderes, das in die Mitte des ägyptischen Bereiches zurücklenkt. Aion, dem der Name des Menes in griechischer Übersetzung zugeordnet wird, zeigt sich in griechischer und römischer Zeit Ägyptens mit Osiris verbunden ${ }^{39}$. Demzufolge wird Mív als der „Osirische“ bezogen. Das ist, von anderer Seite her, wiederum ein Weg des ersten menschlichen Königs auf den mythischen Erstling des ägyptischen Königtums zu ${ }^{10}$. Ob solche Bezüge für oder vielmehr nicht gegen die Historizität des Namensträgers sprechen bzw. was im ganzen daraus zu folgern ist, wird am Schluß erwogen.

Zuvor muß gebührend auf eine andere Namensform hingewiesen werden, die Herodot als der erste griechische Zeuge überhaupt nach der besten Textgestalt ausschließlich verwendet: $M i v$. Vielleicht hat der Umstand, daß der Name des ersten Königs dort nur in casus obliqui vorkommt (Mivv, Mĩva), zu der wahrhaft gröblichen Mißachtung des Textproblems mit seiner lectio difficilior geführt, die der Forscher heute feststellt 41 . Es ist das Geheimnis wissenschaftlicher Einsicht, sich an der richtigen Stelle zu wundern. Wir hoffen, eine solche richtige Stelle getroffen zu haben, wenn wir der auf den ersten Blick verwunderlichen lectio difficilior ernsthaft nachgehen. Dabei darf für die Zeit Herodots eine genaue Scheidung zwischen $\eta$ und $\iota$ im Griechischen ohne weiteres vorausgesetzt werden. Denn die berühmte Wiedergabe des Blökens der Schafe durch $\beta \tilde{\eta} \beta \tilde{\eta}$ liegt nach dem Vater der Geschichtsschreibung und trennt ihn vom späteren Itazismus ab ${ }^{12}$. Hinzu kommt die Differenzierung in Hinsicht der Endung, die keinesfalls als quantité négligeable abgetan werden kann ${ }^{13}$. Damit erhebt sich die Frage, ob man einer Überlieferung Sinn abgewinnen kann, die Min statt des uns gewohnten Menes an die Spitze der ägyptischen Königsreihe setzt. Sie ist unbedenklich mit Ja zu beantworten. Der Gott Min hat für das frühe Königtum eine

\footnotetext{
34 Morenz, Wortspiele in Ägypten, in: Festschr. J. Jahn, 1957, S. $23 \mathrm{ff}$.

35 Ed. Meyer, Ägyptische Chronologie, aus: APAW 1904, Beilage zu S. 125. Später ist aí́vioৎ Beiwort römischer Kaiser: RAC s. v. Aion.

36 RAC s. v. Aion.

37 A. Erman, in: SPAW 1914, (Nr. 9), S. $245 \mathrm{ff}$., vgl. 256, 261, 263. Der Text ist bei Ammianus Marcellinus griechisch überliefert: XVII 4.

38 Ich habe für Hinweise in diesen Angelogenheiten H. Bonnet, E. Edel und W. Müller zu danken.

${ }^{39}$ L. Kákosy, Osiris = Aion, in: Oriens Antiquus 3, 1964, S. 15 ff.; ders., Zu einer Etymologie von Philä: „Insel der Zeit“, in: AAASH 16, 1968, bes. S. 40.

10 Osiris hat durch seine bekannte Rolle eine viel größere „ideologische Realität" als die Mehrzahl seiner Nachfolger und Vorgänger in der Götterdynastie der Listen, denen z. T. nur eine „akademische“ Bedeutung, etwa durch Systemzwang, zukommt.

${ }^{41}$ A. Wiedemann, Herodots zweites Buch mit sachlichen Erläuterungon, 1890, S. 394, hat an einen griechischen Versuch gedacht, den Namen des $M n j$ durch diese Lautung ,ihrem Minos ähnlicher zu gestalten“. Das bleibt eine reine Vermutung, zumal nicht einzusehen ist, warum dem Menes-Minos in diesem Fall die Endung abhanden gekommen sein sollte. Natürlich ist Wiedemann zugute zu halten, daß man den von uns ernst genommenen Gottesnamen „Min" damals meist Chem las, so Wiedemann selbst S. 367 zu Herodot II 91.

42 Kratinos frgm 43; K. war Zeitgenosse des Aristophanes. Die Variante könnte nur im Konsonanten liegen: b oder $\mathrm{m}$ ? Vgl. dazu Varro, De re rustica $2,1,7$.

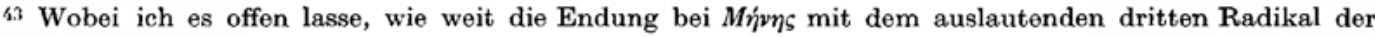
Form $M n j$ zusammenhängt.
} 
bedeutende Rolle gespielt, die man aus den Pyramidentexten längst abgelesen hat `^. Sie läßt sich inzwischen durch manchen Befund absichern und erweitern. So erscheint das Min-Zeichen zwei-

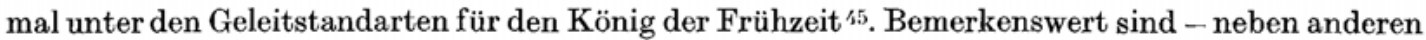
Min-Verbindungen - die Titel $m 3$ t $j \mathbf{M n w}$ und it $M n w$ für einen hohen Funktionär in der dritten Dynastie. Wenn $m 3$ tj als Parallelform zu $m 33$ gefaßt werden darf, würde der Titelträger Hesire „der den Min schaut“ heißen. Das erinnert an den Königinnentitel „die den Horus/Seth schaut“ und könnte im Grenzfall über die Schwelle einer Identifizierung des Königs mit Min führen "16. Wir müssen diese Frage, die gründlicher Untersuchung bedarf und wert ist, hier auf sich beruhen lassen. Das gilt auch für die kaum angepackten historischen Probleme um die drei Minfiguren von Koptos, in denen sich für uns doch wohl nicht ganz zufällig das älteste menschengestaltige Götterbild - dazu gar in Großplastik - darbietet ${ }^{47}$. Wie auch immer aber es sich mit den Anfängen verhalte, der Zusammenhang von Min mit dem Königtum ist in der ägyptischen Geschichte vielfältig bewahrt worden. Man braucht nur an den Gehalt der wertbeständigen Gleichung von Horus und Min und an den Platz des Königtums bei den Minfesten zu erinnern. Die Dinge sind bekannt. Großes Gewicht gewinnt für unser Anliegen der Sachverhalt in der äußersten Spätzeit. Der Horustempel von Edfu enthält, ideologisch und theologisch folgerichtig, eine Kammer des Min ${ }^{18}$. In deren Inschriften heißt der Gott „Ältester des $W n$ - $n f r$, der die beiden Landeshälften $(p s s ̌ t j)$ in Memphis (inbw- $\underline{h} \underline{d} w)$ vereinigt $(d m \underline{d})^{\prime \prime}$ "19. Das liest sich doch wahrhaftig wie eine Aussage über Menes, den Schöpfer des Reiches und Gründer von Memphis. Es ist der Schlußstein in einem Gebäude der Tradition, die es in der Tat verständlich macht, daß Herodot von Min als erstem König Ägyptens spricht, wenn er offenkundig seinen Min auch, gleich Menes, für den ,wirklichen König" hält. Dabei ist leicht.vorzustellen, daß eine allfällige Spannung zwischen dem Gott und dem Menschen auf dem Thron Ägyptens seinerzeit alles andere als unerträglich gewirkt hat. Der letzte König der Götterdynastie war Horus ${ }^{50}$. Warum sollte sich ein aufmerksamer ausländischer Beobachter wundern, wenn Min als Partner des Horus zum ersten König der neuen Reihe wurde, die ja doch trotz ihrer menschlichen Natur auch göttliche Kräfte besaß? Auch lassen sich später Verbindungen zu Menes - Aí́vı „,dem Osirischen“ ziehen. Sie könnten z. B. aus einer Darstellung im Hadrianstor zu Philä abgeleitet werden, wo Min und Osiris im Bilde verschmolzen sind 51. Daraufhin mag man Menes via Osiris „den Minischen“ nennen. Aber all solche Problematik kann getrost beiseite bleiben. Denn sie verleitet zur oberflächlichen Harmonisierung zweier Traditionen, die wir mit Bedacht gesondert haben und beide für sich gelten lassen müssen.

Statt dessen sehen wir zu, wie sich von beiden Traditionen her die Überlieferungsgeschichte ausnimmt. Gleich dem Falle des Cheops ${ }^{52}$ läuft die Sache auch diesmal darauf hinaus, daß man nach dem tatsächlichen Gang der Dinge fragt.

12. 1. 1970

4. Sethe, Urgeschichte und älteste Religion der Ägypter, 1930, § $202 \mathrm{f}$. Mir scheint, diese wichtigen Einsichten Meister Sethes seien sehr zu Unrecht aus unserem Bewußtsein geschwunden, weil das Gesamtkonzept seines Buches keinen Bestand hatte. Für die Beziehung des Min zu den Reichsheiligtümern itrtj und seine Rolle in Memphis vgl. bereits H. Kees, in: ZÄS 57, 1922, bes. S. $128 \mathrm{ff}$.

45 Vgl. z. B. W. Kaiser, Einige Bemerkungen zur ägyptischen Frühzeit, in: ZÄs 84, 1959, bes. S. 122f.

46 Material bei Helck, Beamtentitel S. $45 \mathrm{ff}$.

47 Kunstgeschichtliche Würdigung ("Verbildlichung des Pfeilers") bei W. Wolf, Die Kunst Ägyptens, 1957, S. 57f. Es ist schon verführerisch, im Hinblick darauf wieder einmal den Zusammenhang bei Sethe, Urgeschichte § $202 \mathrm{ff}$. nachzulesen, der - freilich ohne dieser monumentalen Plastik zu gedenken - von einem allfälligen Teilkönigtum in Koptos zu Menes üborgeht. 48 Südlich des zweiten Vorsaales.

49 Rochemonteix, Edfou I 394; vgl. H. Junker, Die Onurislogende, 1917, S. 36 und H. Kees, in: ZÄS 57, 1922, S. 131 mit A. 7. Zu Min und Memphis darf auf die späte Verschmelzung des Min mit PtahSokaris in eine „Ptah-Sokaris“ gonannte Patäkenfigur mit Min-Attributen verwiesen worden: M. Sandman Holmborg, The God Ptah, 1946, S. 183 (nach R. V. Lanzone, Dizionario di Mitologia Egizia, S. 99).

50 So Herodot II 144; Diodor I 44; bei den Listen sind die Verhältnisse komplizierter, vor allem durch die Zwischenschaltung der „Heroen": W. Helek, Untersuchungen zu Manetho und den ägyptischen Königslisten = UGAÄ 18, 1956, bes. S. 4 ff. Dort wird S. 7 auch die Angabe Herodot II 145 diskutiert, daß Pan (= Min) der älteste Gott in Ägypten sei.

51 Kákosy, Etymologie, S. $44 \mathrm{f}$.

52 ZÄS 97, 1971, S. $117 \mathrm{ff}$. 